\title{
Dielectrophoretic classification of fibres: principles and application to glass fibres suspended in air
}

\author{
Frantisek Lizal ${ }^{1, *}$, Milan Maly ${ }^{1}$, Jakub Elcner ${ }^{1}$, Arpad $_{\text {Farkas }}{ }^{1}$, Ondrej Pech $^{1}$, Ondrej Misik ${ }^{1}$, Jan Jedelsky $^{1}$, Miroslav \\ Jicha $^{1}$
}

${ }^{1}$ Brno University of Technology, Faculty of Mechanical Engineering, Energy Institute, Technicka 2896/2, Brno 616 69, Czech Republic

\begin{abstract}
Particles exposed to an electric field experience forces that influence their movement. This effect can be used for filtration of air, or for size classification of aerosols. The motion of charged particles in a non-uniform electric field is called electrophoresis. Two processes are involved in this phenomenon: 1) charging of particles and 2) electrical mobility separation. If fibres are exposed to electrophoresis, they are separated on the basis of two parameters: diameter and length. Regrettably, as naturally occurring fibres are polydisperse both in diameter and length, the electrophoresis is not very efficient in length classification. In contrast, dielectrophoresis is the motion of electrically neutral particles in a non-uniform electric field due to the induced charge separation within the particles. As deposition velocity of fibres induced by dielectrophoretic force strongly depends on length and only weakly on diameter, it can be used for efficient length classification.

Principles of length classification of conducting and non-conducting fibres are presented together with design of a fibre classifier. Lastly, images of motion of fibres recorded by high-speed camera are depicted.
\end{abstract}

\section{Introduction}

The adverse health effects of inhaled fibres are known for decades [1]. As fibre length is believed to be one of the important factors for an assessment of their toxicological impact [2], it is important to be able to produce batches of fibres of identical length for experiments. However, direct manufacture of length-monodisperse fibres and prevention of their breakage during transport and dispersion is extremely challenging. Hence, fibre classifiers are being used for in-situ selecting the fibres of specific length from polydisperse clusters $[2,3]$.

The principles that can be used for length-separation of fibres have been described by Baron [4]. Charged particles exposed to an electric field experience forces that cause motion of the particles. It has been demonstrated by Hochrainer [5] that electrostatically charged longer fibres have higher mobility in a non-uniform electric field compared to short fibres and isometric particles. This fact can be used for the size separation. The principle is called electrophoresis. There are two processes involved in this phenomenon: 1) charging of particles and 2) electrical mobility separation [4]. However, as a result of application of these two processes, the separation is a function of both length and diameter of a fibre. Consequently, classification of fibres based on the principle of electrophoresis is not very efficient [6].
Electrically neutral particles in a non-uniform electric field are also subjected to forces that cause their motion provided they are electrically conductive and the electric field is strong enough to induce charge separation within the particle [4]. This principle is called dielectrophoresis.

The equations for prediction of the deposition velocity $v[\mathrm{~m} / \mathrm{s}]$ in an electric field $E[\mathrm{~V} / \mathrm{m}]$ were derived by Lipowicz and Yeh [7]:

$v=\frac{K_{m} \varepsilon_{0}}{36 \eta} D L\left\{g(\beta)\left(\frac{\alpha}{\alpha-1}-f(\beta)\right)\right\}^{-1} \nabla E^{2}$,

where

$g(\beta)=\frac{8}{3}\left[\frac{-2 \beta}{\beta^{2}-1}+\frac{2 \beta^{2}-1}{\left(\beta^{2}-1\right)^{3 / 2}} \ln \left(\frac{\beta+\left(\beta^{2}-1\right)^{1 / 2}}{\beta-\left(\beta^{2}-1\right)^{1 / 2}}\right)\right]^{-1}$,

And

$$
\begin{aligned}
& f(\beta)=\frac{\beta}{\left(\beta^{2}-1\right)^{1 / 2}} \\
& \times\left[\left(1-\frac{\beta^{2}}{\left(\beta^{2}-1\right)}\right) \operatorname{coth}^{-1}\left(\frac{\beta}{\left(\beta^{2}-1\right)^{1 / 2}}\right)+\frac{\beta}{\left(\beta^{2}-1\right)^{1 / 2}}\right],
\end{aligned}
$$

$K_{m}$ [-] is the dielectric constant of the medium, $\varepsilon_{0}[\mathrm{~F} / \mathrm{m}]$ is the permittivity of free space, $\eta[\mathrm{Pa} \cdot \mathrm{s}]$ is the viscosity of air, $\alpha[-]$ is the ratio of the dielectric constant

\footnotetext{
$\overline{{ }^{*} \text { Corresponding author: lizal@fme.vutbr.cz }}$
} 
of the fibre material to the air, and $\beta[-]$ is the fibre aspect ratio, i.e. the ratio of the length $\mathrm{L}[\mathrm{m}]$ to the diameter $\mathrm{D}[\mathrm{m}]$ of the fibre. For aspect ratios $\beta>5$, the deposition velocity is a function of the length squared, and only a weak function of the diameter [4]. This fact predestines dielectrophoresis as a tool for efficient length classification.

The key factor is the electrical conductivity of a fibre. It has been shown that nonconductive fibres are separated proportionally to the square of the diameter, intermediately conductive fibres depend on both length and diameter. The good length separation is achieved only for conductive fibres with high dielectric constant $[4,7]$. Manmade carbon or silicon carbide fibres are electrically conductive, however, naturally produced fibres are electrical insulators on the macroscopic level [7]. Nonetheless, as for small particles the distance and amount of charge that need to be transported along the fibre is small [8], the microscopic fibres are conductive just due to their size [4]. It has been shown, that sufficient electrical conductivity for the length classification of fibers produced from nonconductive basic material can be achieved by increasing the air humidity [9]. The appropriate relative humidity level is about $50 \%$ [9].

The fibre classifier based on the principle of dielectrophoresis has been developed and tested by Baron et al. [4,10] and Wang et al. [9]. Our team uses the classifier from the Wang's study. We have upgraded the humidifier section and present a method for generation of fibres from glass insulation wool. Also first results of visualization of glass fibre flow in a channel using high-speed camera will be presented.

Our aim is to investigate the fate of inhaled fibres from a common mineral wool intended for thermal insulation. In order to be able to predict the behaviour of fibres in human lungs it is necessary to understand the forces acting on the fibres. The research is divided into two branches. The first branch studies the actual flow of fibres in simple geometries (simple cylinder and single bifurcation) using a high speed camera. Numerical simulations are being performed at the same time, and the results of the actual measurements of the flow of real fibres are being used for validation and refinement of the simulation.

The second branch focuses on deposition of real fibres in a human lung replica. Fibres of various aspect ratios and under several inhalation regimes will be inhaled into the replica and their deposition sites will be evaluated. Again, numerical simulations will be performed simultaneously and their results will be validated by the experiments. Eventually, the results of the first branch, i.e. refined equations for fibre flow in bifurcations, will be used for improvement of the predictions of the deposition in human lungs.

\section{Methods}

An application of the dielectrophoretic classification principles to real glass fibres will be presented in the first part of this chapter, then, the setup of the high speed camera for visualization and recording of the fibre flow will be shown.

\subsection{The fibre classifier setup}

The virgin glass wool Supafil ${ }^{\circledR}$ Loft (Knauf Insulation $\mathrm{GmbH}$, Simbach am Inn, Germany) intended for thermal insulation applied by blowing was disintegrated in a mechanical press to create polydisperse clusters of fibres. Diameters of fibres ranged between 3 and $6 \mu \mathrm{m}$. Before the polydisperse fibres reach the classifier (Figure 1), they need to be properly treated in order to deagglomerate. For this reason, it is necessary to mix the fibres with glass beads (Ballotini impact glass beads, Potters Industries Inc.) and sieve the mixture.

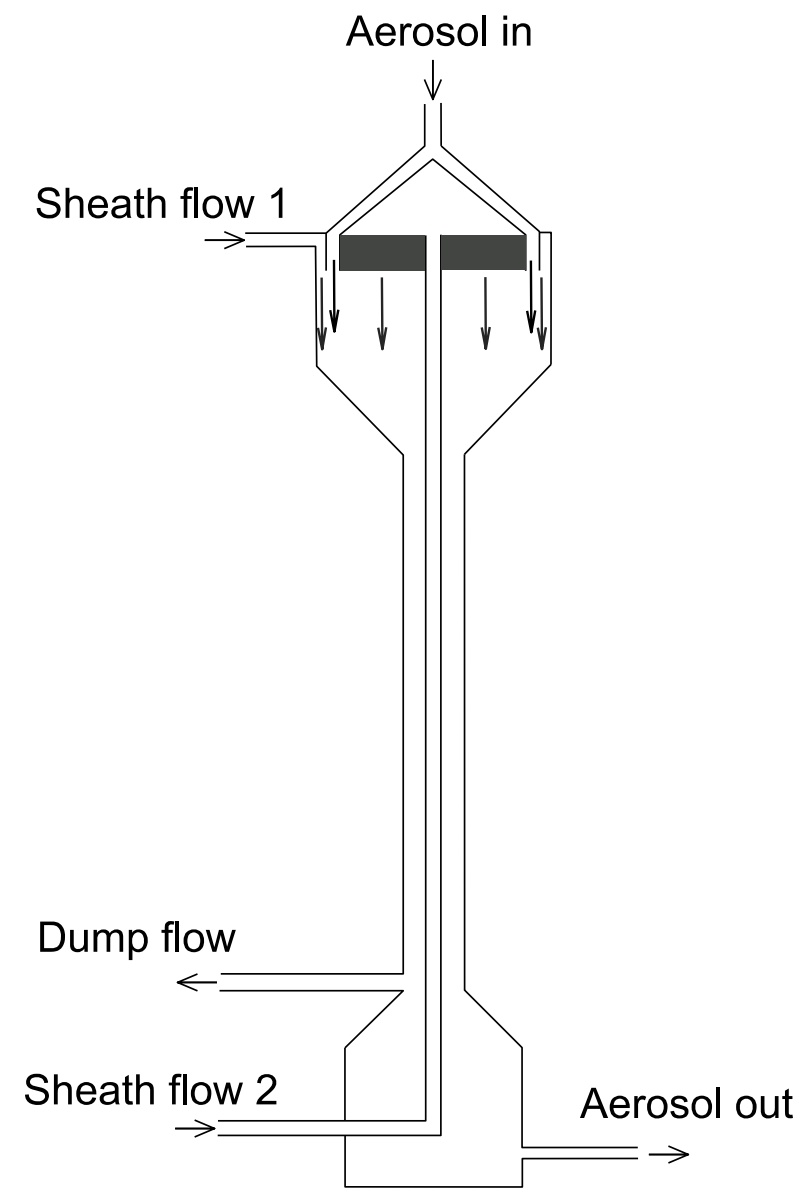

Fig. 1. A scheme of the dielectrophoretic classifier.

Another prerequisite of a successful classification by dielectrophoresis is the electrical conductivity of fibres. In order to ensure the conductivity, it is necessary to control the relative humidity of incoming air. As the compressed air from the laboratory distribution system does not have a guaranteed relative humidity, it was required to incorporate a humidifier into the test rig. As was mentioned earlier, we are using the classifier from Wang's study [9], yet, the original humidifier had to be replaced because of leaks and wear-out.

The newly designed humidification system involves an automatic regulation performed by Arduino - an 
open-source electronic microcontroller (https://www.arduino.cc/). The humidification system consists of a sealed box with one inlet of compressed air from the laboratory distribution system, and one outlet of the humidified air. There are two ultrasonic mist makers (unbranded, country of origin: China, commonly used for application in relaxing garden ponds), which generate very small droplets of water inside the box. As the air passes through the box, it reaches almost $100 \%$ relative humidity. Therefore, to achieve constant and stable level of humidity, it is necessary to mix this air with an air stream of a lower humidity. The mixing is controlled by the Arduino on the basis of the data from a humidity sensor placed downstream. The Arduino operates a servo-controlled valve and regulates the amount of fresh and humidified air in the mixture. The target level of relative humidity is $50 \%$ and the system is able to reach this value with fluctuations of $\pm 2 \%$. The conditioned air is used for all inlets of the classifier, i.e. aerosol flow and both sheath flows (Figure 1).

The second prerequisite of a successful classification by dielectrophoresis is the Gaussian distribution of electrical charge among the fibres. For this purpose, the $\mathrm{Kr}-85$ source of ionising radiation NEKR-10 (Eckert \& Ziegler CESIO, Prague, the Czech Republic) is used.

The path of fibres starts in a hopper, where the mixture of polydisperse fibres with the glass beads is poured. The mixture is slowly transported downstream by a rotary feeder driven by a DC motor geared to slow rotations. Afterwards, it enters a fluidized bed generator, which separates fibres and beads. The beads are send to an overflow vessel, while the fibres are suspended in the humidified air. The fluidized bed is built on the frame of the orbital shaker IKA MS 3 (IKA Werke GMBH \& CO, Staufen, Germany).

Then the fibres flow through the charge equilibrator NEKR-10 and enter the classifier from the top (denoted as the "aerosol in" inlet in the Figure 1). The flow in the classifier is laminar and the fibre aerosol flow is protected by the two sheath flows. The sheath flows apply the humidified air as well. The aerosol flow was set between 1 and $1.5 \mathrm{liter} / \mathrm{min}$, while the sheaths flows were set to 4 liter $/ \mathrm{min}$ each.

Fibres flowing through the annular space in between the inner and outer electrode of the classifier are attracted to the inner electrode, where the highest electric field exists. The fibre aerosol flow is split at the bottom of the classifier, while the narrow length distribution is led to the "aerosol out" output, and the shorter fibres are collected on a filter downstream at the dump flow output. The length of the classified fibres can be controlled by the voltage. Normally the voltage ranges in between 1 and $4 \mathrm{kV}$, while the lower is the voltage, the longer fibres are selected.

\subsection{The high speed camera setup}

A high speed camera Photron SA-Z was used to document the fibre flow inside a straight tube with internal diameter of $15 \mathrm{~mm}$. The flow was illuminated by a pulse LED light model HPLS-36DD18B (Lightspeed
Technologies, USA). The light pulse duration was 300 ns. The camera and light were configured as a Shadowgraph system. The camera frame rate was set to $20,000 \mathrm{fps}$, the frame resolution was $1024 \times 1024 \mathrm{px}$ and the shutter speed was $1 \mu \mathrm{s}$. The long-range microscope lens 12X Zoom (NAVITAR, New York, USA) was used to capture a square section with dimensions of $1.5 \times 1.5 \mathrm{~mm}$, which consequently give a resolution of $1.5 \mu \mathrm{m}$ per pixel.

\section{Results}

Results of both, classification of fibres and recordings of the high speed camera in the simple case of the straight tube are presented in this chapter.

\subsection{The output of fibre classifier}

The original mixture contains polydisperse fibres and also small crumbles of glass pieces as a result of the process of pressing and crushing of the glass wool.

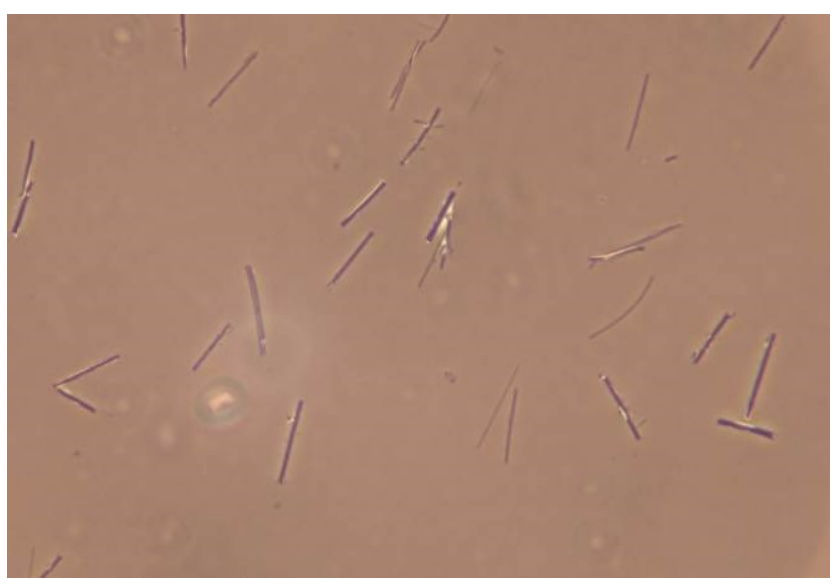

Fig. 2. Example photograph of the classified glass fibres

As can be seen in Figure 2, most of the impurities were cleared away and finished in the dump flow (Figure 3 ).

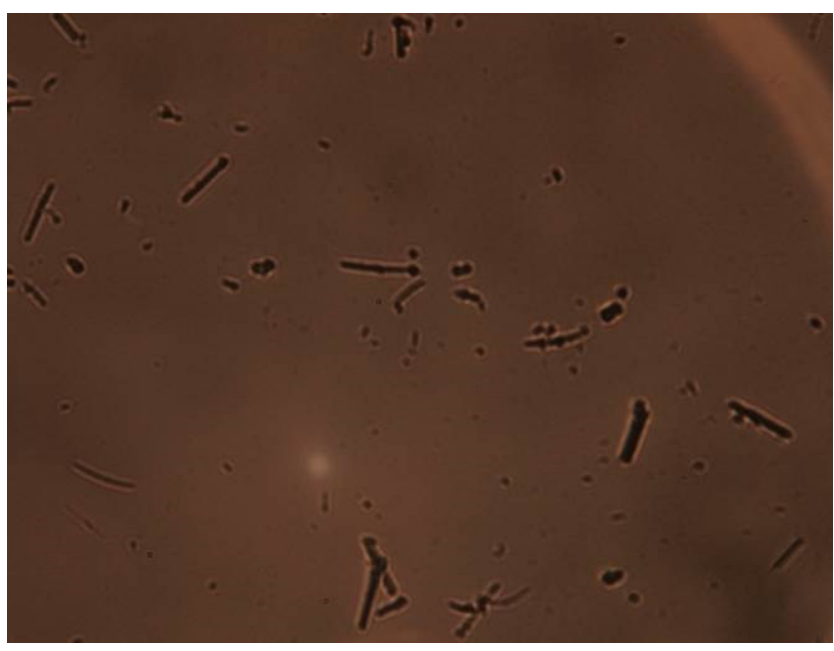

Fig. 3. Example photograph of the glass fibres collected on a dump flow filter 
The fibres longer than the classified fraction stayed inside the classifier at the inner rod, which has been treated with a thin homogenous layer of grease to prevent bouncing of the fibres. Hence, all fibres that touched the surface of the inner rod stuck there.

The experiments proved that the automated humidifier has improved the quality of the classified fibres and simplified the operation of the whole experimental rig. Also, the water pump, which was a part of the original design, was no longer needed.

However, it has to be noted, that longer operation of the classifier requires frequent interventions of the operator and continuous supervision, as there are several critical components of the system, which can cause malfunction of the classifier. These are mainly the feeder line from the hopper, which is susceptible to congestions, and the fluidized bed with the tubes connected to it which suffer problems due to vibrations of the orbital shaker. Also the glass beads overflow and the electrical circuit has to be regularly checked. The whole classifier has to be disassembled and cleaned after several hours of operation.

Also, it has to be noted, that the larger were the diameters of the fibres, the worse was the quality of the length separation.

\subsection{The results of high speed camera visualization}

The high speed camera recordings were analysed and fibres were clearly detected (Figure 4). As a next step, the tracking of fibres will be automated and angles of fibres in selected positions within the tube will be statistically evaluated to get data for validation of numerical simulations, which will be performed on the same geometry.

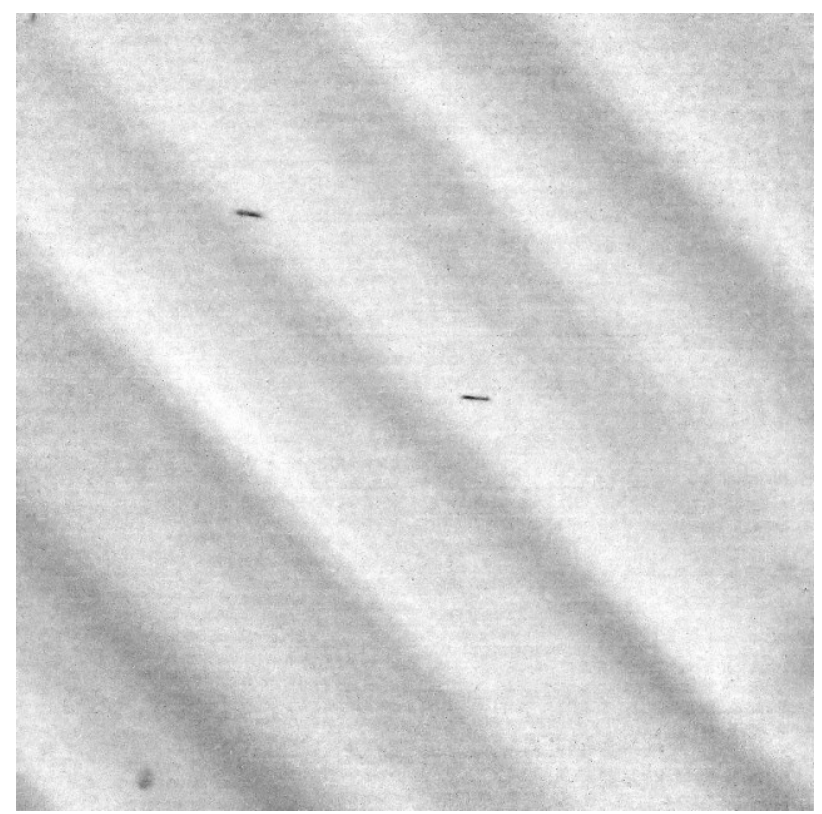

Fig. 4. A sample image from the high speed camera recording.

\section{Conclusions}

The dielectrophoretic classifier was upgraded with the automatic humidification system working on the basis of the Arduino microcontroller board. The newly designed humidifier simplified the operation of the classifier and proved to be capable of long-term operation. Nonetheless, despite this simplification, the operation of the classifier still needs continuous supervision and regular checks of the critical components mentioned earlier in this paper.

The classifier produced classified fibres in desired lengths, however, better results were achieved for smaller diameters of fibres.

The experiments proved that the high speed camera is capable of recording the glass fibres flowing in the cylindrical tube with diameter similar to the human trachea. The recordings are suitable for further analysis using methods of image processing, and will be used for validation and refinement of numerical simulations.

Acknowledgement: This work was supported by the Czech Science Foundation under the grant GA18-25618S, and by the project FSI-S-17-4444.

\section{References}

1 K. R. Spurny, H. Opiela, G. Weiss, and W. Stober, American Industrial Hygiene Association Journal 40 (1), 20 (1979); M. Lippmann, Environ Res 46 (1), 86 (1988).

2 B. K. Ku, G. Deye, and L. A. Turkevich, Journal of Aerosol Science 114, 250 (2017).

3 Z. Wang, P. K. Hopke, G. Ahmadi, Y. S. Cheng, and P. A. Baron, Journal of Aerosol Science 39 (12), 1040 (2008).

4 P. A. Baron, G. J. Deye, and J. Fernback, Aerosol Science and Technology 21 (2), 179 (1994).

5 D. Hochrainer, G. Zebel, and V. Prodi, Staub Reinhalt Luft 38 (10), 425 (1978).

6 R. J. Han and J. W. Gentry, Journal of Aerosol Science 24 (2), 211 (1993).

7 P. J. Lipowicz and H. C. Yeh, Aerosol Science and Technology 11 (3), 206 (1989).

8 N. A. Fuks, The mechanics of aerosols, Rev. and enl. ed. (Macmillan, New York,, 1964).

9 Z. C. Wang, P. K. Hopke, P. A. Baron, G. Ahmadi, Y. S. Cheng, G. Deye, and W. C. Su, Aerosol Science and Technology 39 (11), 1056 (2005).

10 G. J. Deye, P. Gao, P. A. Baron, and J. Fernback, Aerosol Science and Technology 30 (5), 420 (1999). 